\title{
LOOMING AUDITORY AND VIBROTACTILE COLLISION WARNINGS FOR SAFE DRIVING
}

\author{
Cristy $\mathrm{Ho}^{1}$, Charles Spence ${ }^{1} \&$ Rob Gray ${ }^{2}$ \\ ${ }^{1}$ Crossmodal Research Laboratory, University of Oxford, Oxford, UK \\ ${ }^{2}$ School of Sport and Exercise Sciences, University of Birmingham, Birmingham, UK \\ Email: cristy.ho@psy.ox.ac.uk
}

\begin{abstract}
Summary: Looming auditory warning signals (that is, signals whose intensity increases over time) have proven to be particularly effective in terms of reducing a driver's brake reaction times (BRTs) to impending collisions, and are also associated with very low false alarm rates. We report two experiments designed to further investigate how the presentation of looming auditory warnings with increasing frequency or increasing spatial extent would compare to those with increasing intensity. A third experiment was conducted in order to evaluate the potential efficacy of presenting looming warnings to drivers in another modality, namely via vibrotactile signals. Participants' speeded BRTs to potential collision events following the presentation of various warning signals in a simulated car following scenario were measured. While both looming frequency and spatial warnings were effective in terms of speeding the driver's responses to critical driving events, the magnitude of the benefit resembled that of a typical nonlooming constant intensity warning. Looming intensity warnings outperformed their looming frequency counterparts in terms of facilitating drivers' collision avoidance responses. As for vibrotactile warnings, the results revealed that looming vibrotactile stimuli did not offer any additional benefits over and above the other non-looming vibrations tested in the study. The implications of these findings for collision warning systems design are discussed.
\end{abstract}

\section{INTRODUCTION}

The surge of interest in neuroergonomics for car interface design over the last decade supports the view that the future design of cars should be centered on the human brain (and its limitations in terms of information processing; Spence, 2012). In particular, recent empirical evidence suggests that the presentation of non-visual warning signals in a driver's peripersonal space (i.e., close to, or on, the driver's body) may represent a unique means of communicating behaviorally relevant stimuli that demand the driver's immediate attention (see Ho \& Spence, 2009). Many researchers have focused their efforts on trying to identify specific properties of warning signals that define their effectiveness in terms of eliciting speeded and appropriate behavioral responses from drivers for collision avoidance.

One interesting area concerns the investigation of the relative effectiveness of those warning signals whose intrinsic properties potentially convey time-to-collision (TTC; i.e., speed of approach) information to drivers. To this end, Gray (2011) recently demonstrated that auditory looming warnings might facilitate a driver's speeded collision avoidance responses. In his original driving simulator study, drivers' brake reaction times (BRTs) to potential collisions in a car following scenario were found to be significantly shorter when auditory looming warnings 
whose intensity increased as a function of the driver's speed were presented, as compared to those conditions in which the auditory warnings had a constant intensity, a ramped intensity that increased continuously, a pulsed signal that went on and off repeatedly, or else when no warning was presented. Notably, while the looming auditory warnings were just as effective as the symbolic car horn warnings in term of reducing BRTs, significantly fewer false alarm responses to unreliable warnings (i.e., catch trials) were observed. These findings suggest that auditory looming warnings might be used to convey TTC and/or urgency information that is inherent in the signals themselves (see also Shaw, McGowan, \& Turvey, 1991).

Given that Gray (2011) only tested auditory looming warnings of increasing sound intensity, the purpose of the present study was to further investigate the looming dimension that contributes to any potential performance advantage for drivers. Specifically, the idea was to examine whether approach information could be conveyed to drivers in another way. For instance, it has been proposed that changes in auditory frequency could be used for collision warnings. In particular, frequency information could be used to warn pedestrians about approaching electric cars without adding noise pollution (Changizi, 2012). In Experiment 1, the efficacy of looming frequency warnings was evaluated by comparing them to looming intensity warnings. Meanwhile, as looming visual signals equate to increasing visual size on the retina, Experiment 2 was designed to examine whether such spatial expansion information could be expressed in the form of spatially expanding auditory signals. Experiment 3 was designed to extend the study from the auditory domain to the tactile modality. There we investigated whether looming vibrations might offer an alternative to auditory looming. Such an approach seemed appropriate given the growing trend to install vibrotactile warning systems in next generation cars (e.g., López, 2012).

\section{METHODS}

A simulated driving task modelling a car following scenario was utilized in the present study. Participants' speeded BRTs to potential collision events were measured. Experiments 1 and 2 were conducted using the same driving simulator setup as reported in Gray (2011). The only exception was that in Experiment 2, a nine-speaker array positioned inside the vehicle dashboard was used to present the spatial looming signals. Experiment 3 was conducted in the simulated driving setup used by Ho and Spence (2009, Experiment 3), with $60 \mathrm{~Hz}$ screen refresh rate. Thus, the interval between the onset of the fast closing-in on the lead car and collision was $1900 \mathrm{~ms}$.

\section{Participants}

Twelve naïve participants took part in each of the experiments (see Table 1). All of the participants reported normal or corrected-to-normal vision, and normal hearing (for Experiments 1 and 2) or a normal sense of touch (for Experiment 3). They all completed an informed consent form and were compensated for their participation.

Table 1. Driver characteristics

\begin{tabular}{ccccccc}
\hline \multirow{2}{*}{ Experiment } & \multicolumn{2}{c}{ Gender } & \multicolumn{2}{c}{ Age (years) } & \multicolumn{2}{c}{ Driving experience (years) } \\
& Women & Men & Mean & Range & Mean & Range \\
\hline 1 & 6 & 6 & 23.1 & $21-29$ & 5.1 & $3-9$ \\
2 & 5 & 7 & 24.5 & $20-31$ & 4.6 & $2-8$ \\
3 & 8 & 4 & 26.1 & $20-32$ & 5.7 & $0.5-12$ \\
\hline
\end{tabular}


Table 2. Warning signal conditions presented in the present study

\begin{tabular}{|c|c|c|c|c|c|}
\hline \multirow{2}{*}{ Experiment } & \multicolumn{5}{|c|}{ Warning signal conditions } \\
\hline & (a) & (b) & (c) & (d) & (e) \\
\hline 1 & $\begin{array}{c}\text { No } \\
\text { warning }\end{array}$ & $\begin{array}{l}\text { Constant intensity } \\
\text { tone }\end{array}$ & $\begin{array}{l}\text { Looming intensity } \\
\text { tone }\end{array}$ & $\begin{array}{l}\text { Looming frequency } \\
\text { tone }\end{array}$ & $(\mathrm{c})+(\mathrm{d})$ \\
\hline 2 & $\begin{array}{c}\text { No } \\
\text { warning }\end{array}$ & $\begin{array}{l}\text { Constant intensity } \\
\text { white noise }\end{array}$ & $\begin{array}{l}\text { Looming intensity } \\
\text { white noise }\end{array}$ & $\begin{array}{l}\text { Looming spatial } \\
\text { white noise }\end{array}$ & $(c)+(d)$ \\
\hline 3 & $\begin{array}{c}\text { No } \\
\text { warning }\end{array}$ & $\begin{array}{l}\text { Constant intensity } \\
\text { vibrations }\end{array}$ & Ramped vibrations & Looming vibrations & $\begin{array}{c}\text { Pulsed } \\
\text { vibrations }\end{array}$ \\
\hline
\end{tabular}

\section{Warning signals}

Experiment 1. Table 2 describes the different warning signal conditions presented in the present study. All of the auditory stimuli were $1000 \mathrm{~ms}$ in duration. The constant intensity warnings consisted of a $2000 \mathrm{~Hz}$ tone presented at $75 \mathrm{~dB}$. The looming intensity warnings were identical to those presented in Gray (2011), with an intensity that increased from about $60 \mathrm{~dB}$ at the simulated distance of $100 \mathrm{~m}$ up to a maximum of $85 \mathrm{~dB}$. The looming frequency warnings had an initial frequency of $2000 \mathrm{~Hz}$, and the frequency $(F)$ was modified according to $F_{w} \approx a+k D^{-2}$ where the value of $D$ at each instant was determined by the driver's speed at the onset of the warning. Values of $a=1000$ and $k=10^{7}$ were chosen so that the frequency of warning signals ranged between roughly $2000-5000 \mathrm{~Hz}$.

Experiment 2. All of the auditory stimuli were $1000 \mathrm{~ms}$ in duration. The constant intensity warnings consisted of white noise presented at $75 \mathrm{~dB}$. The looming intensity warnings were the same as those used in Gray (2011), except that white noise rather than a pure tone was used. The looming spatial warnings consisted of uncorrelated white noise presented from each loudspeaker in the nine-speaker array (see Figure 1). The output levels were balanced for each configuration so that the total intensity, as measured from the driver's position, was $75 \mathrm{~dB}$. The angular size of the looming spatial warning signal was changed according to $\theta=\operatorname{atan}(W / D)$ where $W$ was the width of the simulated lead vehicle $(1.8 \mathrm{~m})$ and $D$ was its distance. The distance of the lead vehicle at the onset of the warning was determined by $D_{w}=T T C_{\text {thres }} \times d D / d t+S P \times V_{F}$ where $d D / d t$ was the closure rate, and $V_{F}$ was the driven vehicle's speed. The values for $T T C_{\text {thres }}$ (time-to-collision threshold) and $S P$ (speed penalty) were identical to that used in Gray (2011). As illustrated in Figure 1, signal $t_{1}$ was presented at $D_{w}$, and signal $t_{2}$ was presented when TTC was 500 ms less than the TTC value at warning onset. Signal $t_{3}$ was presented when TTC fell below $2 \mathrm{~s}$. Thus, the rate of increase in spatial extent of the auditory signal was directly related to the closure rate.

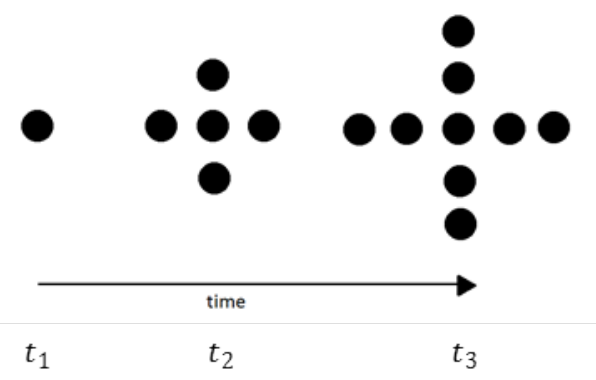

Figure 1. Configuration of looming spatial speakers utilized in Experiment 2 
Experiment 3. All of the vibrotactile stimuli were delivered at a frequency of $250 \mathrm{~Hz}$. The constant intensity warnings had an intensity that was half of the full intensity of the tactor. The ramped and looming warnings both began at two-ninths of the full intensity of the tactor and ended at seven-ninths of the full intensity. The ramped warnings increased at a fixed rate continuously. For the looming warnings, the intensity $(I)$ was updated according to the formula $I \approx a+k(T / 250)^{2}$ where $a$ was the initial intensity and $T$ was the time (in ms) from the onset of the warning. The value of $k=2.6$ was chosen to make the looming warnings end at the same intensity level as that of the ramped warnings. The pulsed warnings were turned on to half of the full intensity and off (i.e., zero intensity) once every 100 ms.

\section{Design and procedure}

Experiments 1 and 2. Apart from the warning signal conditions (see Table 2), the design and procedure for Experiments 1 and 2 were identical to those reported in Gray (2011).

Experiment 3. In this 25-minutes experiment, only the forward head position condition (see Ho \& Spence, 2009) was presented. The participants had to depress the brake pedal as rapidly as possible whenever they detected the sudden closing-in on the lead car in the video clips. The experimental session consisted of a block of 12 practice trials and four blocks of 72 experimental trials. The four critical warning signal conditions were randomly chosen and presented equiprobably within each block of experimental trials. Catch trials were presented at a ratio of $1: 4$ to that of the critical trials (thus rendering the reliability of the warnings as $80 \%$ predictive, cf. Ho, Reed, \& Spence, 2006). Baseline trials in which no warning signal preceded the sudden closing-in on the lead car were also presented at a ratio of 1:4 to that of the critical trials. The presentation of a warning signal coincided with the sudden fast closing-in on the lead car, and was terminated $900 \mathrm{~ms}$ after its onset or after a participant's response, whichever occurred first.

\section{RESULTS}

Mean BRT data, defined as the elapsed time between the onset of the collision warning and the time at which a participant initiated their braking response by depressing the brake pedal, were analysed using a one-way repeated measures ANOVA with the within-participants factor of Warning Signal. Post hoc Tukey's Tests were then performed in order to determine the relative effectiveness of each collision warning against the others.

\section{Experiment 1}

The analysis on the BRT data from Experiment 1 revealed a significant main effect of Warning Signal, $F(4,44)=19.0, p<.001$ (see Figure 2). In particular, post hoc pairwise comparisons revealed that participants responded significantly more rapidly following the presentation of Looming Intensity, Looming Frequency, and Looming Intensity + Frequency warnings than when no warning was presented (see Table 3). Importantly, both Looming Intensity and Looming Intensity + Frequency warnings gave rise to a significant performance advantage (i.e., shorter BRT by over $100 \mathrm{~ms}$ ) over the Constant Intensity and Looming Frequency warnings. The pairwise differences between No Warning and Constant Intensity, Constant Intensity and Looming Frequency, and Looming Intensity and Looming Intensity + Frequency failed to reach statistical significance. 

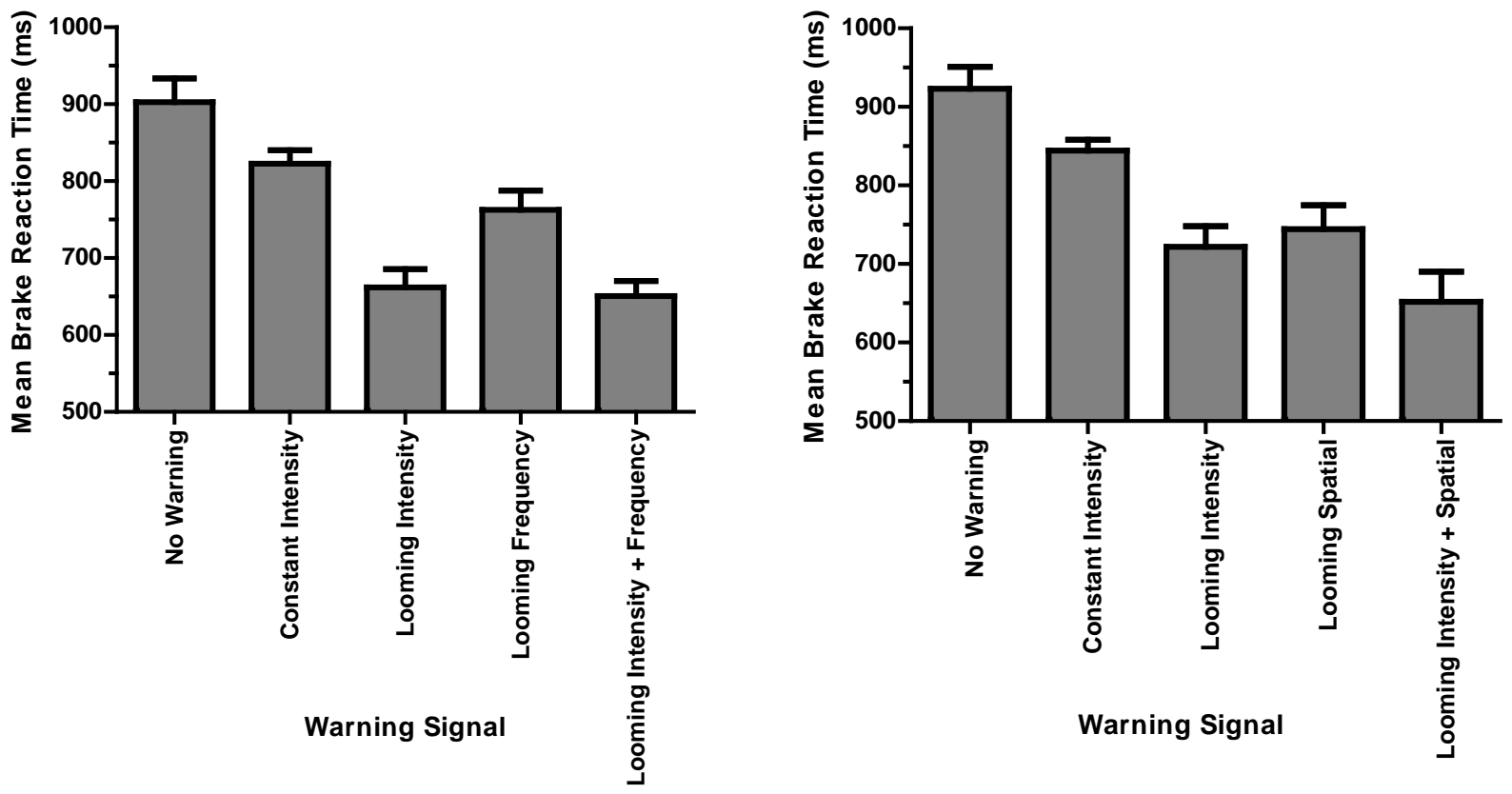

Figure 2. Mean BRT (in ms) of participants' braking responses a function of the Warning Signal in Experiments 1 (left) and 2 (right). Error bars indicate the standard errors of the means

Table 3. Pairwise comparisons from Tukey's Test in Experiments 1 (left) and 2 (right) Mean differences (in ms) between the different warning signals are shown. Significant comparisons $* \mathrm{p}<.05,{ }^{* *} \mathrm{p}<.01$, and ${ }^{* *} \mathrm{p}<.001$ are indicated

\begin{tabular}{|c|c|c|c|c|c|c|c|c|c|}
\hline $\begin{array}{c}\text { Warning } \\
\text { Signal }\end{array}$ & $\begin{array}{l}\text { Constant } \\
\text { Intensity }\end{array}$ & $\begin{array}{l}\text { Looming } \\
\text { Intensity }\end{array}$ & $\begin{array}{l}\text { Looming } \\
\text { Frequency }\end{array}$ & $\begin{array}{l}\text { Looming } \\
\text { Intensity+ } \\
\text { Frequency }\end{array}$ & $\begin{array}{c}\text { Warning } \\
\text { Signal }\end{array}$ & $\begin{array}{l}\text { Constant } \\
\text { Intensity }\end{array}$ & $\begin{array}{l}\text { Looming } \\
\text { Intensity }\end{array}$ & $\begin{array}{c}\text { Looming } \\
\text { Spatial }\end{array}$ & $\begin{array}{l}\text { Looming } \\
\text { Intensity } \\
+ \text { Spatial } \\
\end{array}$ \\
\hline $\begin{array}{c}\text { No } \\
\text { Warning }\end{array}$ & 80 & $241^{* * *}$ & $140 * *$ & $252 * * *$ & $\begin{array}{c}\text { No } \\
\text { Warning }\end{array}$ & 79 & $202 * * *$ & $179 * * *$ & $272 * * *$ \\
\hline $\begin{array}{l}\text { Constant } \\
\text { Intensity }\end{array}$ & & $161^{* * *}$ & 60 & $172 * * *$ & $\begin{array}{l}\text { Constant } \\
\text { Intensity }\end{array}$ & & $123 *$ & 100 & $193 * * *$ \\
\hline $\begin{array}{l}\text { Looming } \\
\text { Intensity }\end{array}$ & & & $-101^{*}$ & 11 & $\begin{array}{l}\text { Looming } \\
\text { Intensity }\end{array}$ & & & -23 & 70 \\
\hline $\begin{array}{l}\text { Looming } \\
\text { Frequency }\end{array}$ & & & & $112 *$ & $\begin{array}{c}\text { Looming } \\
\text { Spatial }\end{array}$ & & & & 93 \\
\hline
\end{tabular}

\section{Experiment 2}

Similar analyses performed on the BRT data from Experiment 2 again revealed a significant main effect of Warning Signal, $F(4,44)=14.1, p<.001$ (see Figure 2). Post hoc Tukey's Test (see Table 3) revealed that participants’ BRTs were significantly shorter following the presentation of Looming Intensity, Looming Spatial, and Looming Intensity + Spatial warnings than when no warning signal was presented. The presentation of both Looming Intensity and Looming Intensity + Spatial warnings gave rise to significantly faster braking responses ( $>120$ ms facilitation) by participants than when Constant Intensity warnings were presented. None of the other pairwise comparisons reached statistical significance. 


\section{Experiment 3}

RTs falling 2.5 standard deviations above or below the mean RT for a particular condition for each participant were discarded from the data analysis. On average, $2.2 \%$ of the trials were removed. Similar analyses on the BRT data from Experiment 3 revealed a significant main effect of Warning Signal, $F(4,44)=16.0, p<.001$ (see Figure 3). In particular, post hoc comparisons revealed significant difference between all four warning types with the no warning condition (all $p<.001$; see Table 4). This suggests that participants responded more rapidly following the presentation of Constant Intensity warnings, Ramped warnings, Looming warnings, or Pulsed warnings than when no vibration was presented. None of the pairwise comparisons among the four warning signals reached statistical significance. Overall, participants responded to $10.4 \%$ of the catch trials on average ( $S E=4.3 \%)$.

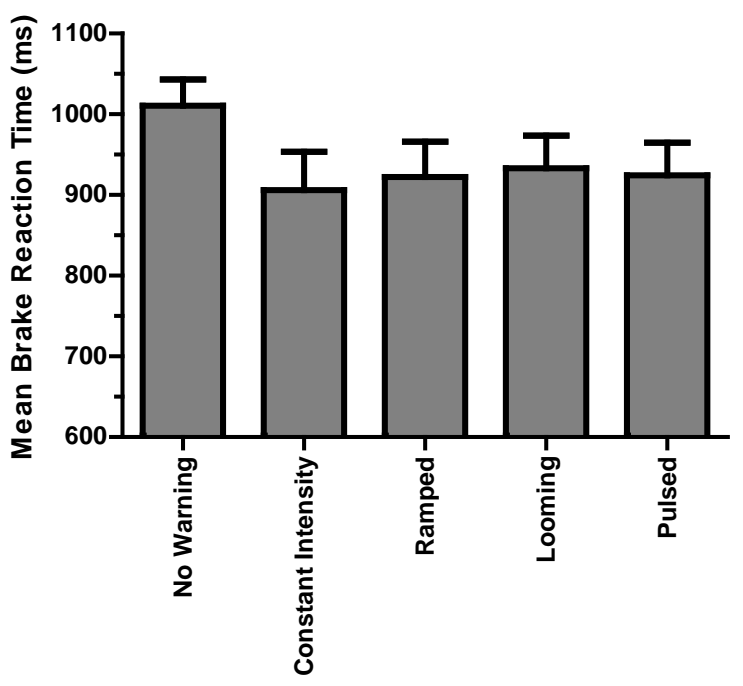

Warning Signal

Table 4. Pairwise comparisons from Tukey's Test in Experiment 3. Mean differences (in ms) between the different warning signals are shown. Significant comparisons $* * * \mathbf{p}<.001$ are indicated

\begin{tabular}{ccccc}
\hline $\begin{array}{c}\text { Warning } \\
\text { Signal }\end{array}$ & $\begin{array}{c}\text { Constant } \\
\text { Intensity }\end{array}$ & Ramped & Looming & Pulsed \\
\hline $\begin{array}{c}\text { No } \\
\text { Warning }\end{array}$ & $104^{* * *}$ & $88^{* * *}$ & $77^{* * *}$ & $86^{* * *}$ \\
$\begin{array}{c}\text { Constant } \\
\text { Intensity }\end{array}$ & & -16 & -27 & -18 \\
Ramped & & -11 & -2 \\
& & & \\
Looming & & & & 9
\end{tabular}

Figure 3. Mean BRT (in ms) of participants' braking responses a function of the Warning Signal in Experiment 3. Error bars indicate the standard errors of the means

\section{CONCLUSIONS}

The findings from the first two experiments suggest that auditory looming intensity warnings outperform other forms of auditory looming signals in terms of facilitating a driver's speeded collision avoidance responses. It is possible that looming intensity warnings convey some sort of perceptual and behavioral salience that is not observable in looming frequency and looming spatial warnings. Importantly, when different looming warnings were combined together, the effectiveness of the looming intensity warnings still remained. Given that sound intensity, while being a prominent cue, is not a definite cue for distance (see Hall \& Moore, 2003), it may be advantageous to encode plausibly redundant looming information via multiple sound properties.

Contrary to our hypothesis, however, the effectiveness of the vibrotactile looming intensity warnings did not stand out from the other non-looming vibrotactile warnings tested (either in 
terms of BRT or in terms of a reduction in false alarm rates). Given that in Experiment 3 the rate of closing-in on the lead car was kept constant, it is possible that vibrotactile looming intensity warnings may turn out to be more effective than non-looming vibrations under conditions when closure rate is variable (i.e., when TTC information is critical for the initiation of the appropriate collision avoidance responses). This will require running the experiment in a high fidelity driving simulator setup such as the one used in the first two experiments.

Taken together, the present findings clearly suggest that auditory looming warnings that increase in intensity as a function of TTC represent a particularly promising means of alerting a driver and redirecting their spatial attention for immediate safety-critical reactions. The presentation of looming intensity information, whether via auditory or vibrotactile signals, allows warning signals to be progressively presented at an initially less intrusive manner (but of a level that is sufficient to alert and prepare a driver to make the appropriate response) while maintaining a low false alarm response to the no collision situation. This is especially crucial as inappropriate driver responses to unreliable signals may result in undesirable consequences (e.g., mistrust). Future studies can examine the feasibility of extending the current idea to present looming signals to convey distance information in other in-car navigation and warning systems.

\section{ACKNOWLEDGMENTS}

This research was supported in part by a grant (EP/J008001/1) from the EPSRC.

\section{REFERENCES}

Changizi, M. (2012, September 13). For stealthy electric cars, auditory illusions could save lives. Discover Magazine. Retrieved October 29, 2012 from http://blogs.discovermagazine.com/ crux/2012/09/13/for-stealthy-electric-cars-auditory-illusions-could-save-lives/

Gray, R. (2011). Looming auditory collision warnings for driving. Human Factors, 53, 63-74.

Hall, D. A., \& Moore, D. R. (2003). Auditory neuroscience: The salience of looming sounds. Current Biology, 13, R91-R93.

Ho, C., Reed, N., \& Spence, C. (2006). Assessing the effectiveness of "intuitive" vibrotactile warning signals in preventing front-to-rear-end collisions in a driving simulator. Accident Analysis \& Prevention, 38, 989-996.

Ho, C., \& Spence, C. (2009). Using peripersonal warning signals to orient a driver's gaze. Human Factors, 51, 539-556.

López, J. A. (2012, July 18). Kia named Quoris his K9 flagship sedan for export markets. Retrieved October 11, 2012, from http://thekoreancarblog.com/2012/07/kia-named-quorisk9-flagship-sedan-export-markets-wvideos/

Shaw, B. K., McGowan, R. S., \& Turvey, M. T. (1991). An acoustic variable specifying time-tocontact. Ecological Psychology, 3, 253-261.

Spence, C. (2012). Drive safely with neuroergonomics. The Psychologist, 25, 664-667. 